\title{
Sustained long-term benefits of patient support program participation in immune-mediated diseases: improved medication-taking behavior and lower risk of a hospital visit
}

\author{
A Mark Fendrick, MD; Diana Brixner, PhD; David T Rubin, MD; Philip Mease, MD; Harry Liu, PhD; Matthew Davis, MA; \\ and Manish Mittal, PhD
}

\author{
What is already known \\ about this subject \\ - Patient support programs (PSPs) \\ improve medication-taking behavior \\ in the first 12 months of treatment \\ for patients with immune-mediated \\ diseases. \\ - Improved disease management \\ through better medication-taking \\ behavior and PSP support should \\ lower the need for hospital visits, \\ which incur substantial burden for \\ patients and payers. \\ - PSPs aim to improve long-term \\ outcomes for patients with immune- \\ mediated diseases by addressing \\ barriers to optimal adherence; \\ however, there is a paucity of \\ evidence due to the novelty of \\ multifaceted PSPs and the rarity of \\ important clinical events such as \\ hospital visits.
}

\author{
What this study adds \\ - This study used real-world data to show \\ that participation in the evaluated \\ PSP is associated with sustained \\ improvements in medication-taking \\ behavior and lower risk of hospital visits \\ over 36 months. \\ - These findings provide further evidence \\ that PSP participation can improve \\ patient outcomes while also lowering \\ the burden to the health care system \\ over the longer term.
}

\author{
Author affiliations \\ A Mark Fendrick, MD, University of Michigan, \\ Ann Arbor, Diana Brixner, PhD, University of \\ Utah College of Pharmacy, Salt Lake City, \\ David T Rubin, MD, University of Chicago \\ Medicine Inflammatory Bowel Disease \\ Center, Chicago, IL; Philip Mease, MD, \\ Swedish Medical Center/Providence St. \\ Joseph Health and University of Washington \\ School of Medicine, Seattle, WA; Harry \\ Liu, PhD, RAND Corporation, Boston, MA; \\ Matthew Davis, MA, Medicus Economics, \\ LLC, Milton, MA; and Manish Mittal, PhD, \\ AbbVie Inc., North Chicago, IL. \\ AUTHOR CORRESPONDENCE: \\ Manish Mittal, 847.935.9190; \\ manish.mittal@abbvie.com
}

J Manag Care Spec Pharm. 2021;27(8):1086-95

Copyright $@ 2021$, Academy of Managed Care Pharmacy. All rights reserved.

long-term medication-taking behavior, and hospital visits.

long-term treatment plan and avoid costly hospital visits is crucial. Launched nationally in 2015, HUMIRA Complete (a PSP for adalimumab patients) provides an opportunity to study long-term effects of PSP participation, including the impact on medication-taking behavior and hospital visits.

OBJECTIVE: To evaluate the sustained relationship between PSP participation,
METHODS: A longitudinal, retrospective matched-cohort study was conducted of patients initiating adalimumab between January 2015 and February 2016 with or without enrolling in the PSP, using patientlevel data from the HUMIRA Complete PSP linked with Symphony Health claims. The sample included adult, commercially insured 
patients diagnosed with an indicated disease who were biologicnaive and had data available for $\geq 6$ months before and $\geq 12$ months after initiating adalimumab. Adherence (proportion of days covered) and hospital visits were assessed at 12, 24, and 36 months for patients with sufficient follow-up data. Multivariable generalized models estimated differences between cohorts, controlling for baseline characteristics and hospital visits. Duration of persistence and time to a hospital visit were compared using Kaplan-Meier analyses. Hazard ratios were estimated using multivariable Cox proportional hazards models.

RESULTS: The matched cohort included 2,268 patients ( 1,134 per cohort), and patient attrition was similar across cohorts. The PSP cohort consistently demonstrated higher adalimumab adherence than the non-PSP cohort at $12(64.8 \%$ vs. $50.1 \%, P<0.0001 ; 29 \%$ greater), 24 (49.4\% vs. $38.4 \% ; P<0.0001 ; 29 \%$ greater), and $36(39.4 \%$ vs. $35.1 \% ; P=0.02 ; 12 \%$ greater) months. PSP participation was associated with a $30 \%$ lower hazard of discontinuation $(P<0.0001)$, and median duration of persistence was 4.8 months longer for the PSP cohort (13.2 vs. 8.4 months; $P<0.0001$ ). The PSP cohort had lower rates of hospital visits at 12 (30\% vs. 37\%; $P<0.001 ; 19 \%$ lower), 24 (44\% vs. $53 \% ; P=0.01 ; 17 \%$ lower), and 36 (55\% vs. $65 \% ; P<0.01 ; 16 \%$ lower) months, and PSP participation was associated with a $25 \%$ lower hazard of a hospital visit $(P<0.0001)$. Median time to a hospital visit was 10.8 months longer for the PSP cohort (32.7 vs. 21.9 months; $P<0.0001)$. Findings were consistent across therapeutic areas: hazard of a hospital visit was $28 \%, 27 \%$, and $37 \%$ lower for rheumatology, gastroenterology, and dermatology patients participating in the PSP (all $P<0.05$ ).

CONCLUSIONS: Patients with immune-mediated diseases receiving adalimumab and utilizing this PSP had improved long-term medication-taking behavior and lower risk of hospital visits, demonstrating the potential of PSPs to improve patient outcomes and lower the burden to the health care system.

For patients with immune-mediated diseases, participation in a patient support program (PSP) can augment value-based care by helping patients adhere to complex treatment regimens. ${ }^{1-3}$ Better medication-taking behavior (adherence and persistence) with biologic therapy is associated with improved clinical, economic, and humanistic outcomes; however, suboptimal adherence remains common. ${ }^{3-5}$ Nonadherence exacerbates disease progression and increases the risk of hospitalization. ${ }^{6-11}$ Though PSP use has been consistently associated with improved outcomes for patients initiating treatment, there is a paucity of evidence on (a) whether medication-taking behavior benefits are sustained throughout treatment due to the novelty of multifaceted PSPs and (b) the relationship between PSP participation and hospital visits due to rarity. ${ }^{1-3,12-14}$ As immune-mediated diseases continue to rapidly increase in prevalence and economic burden, understanding the potential sustained value of PSP participation in helping patients adhere to their long-term treatment plan and avoid hospital visits should be of great interest to patients, providers, and payers. ${ }^{15-17}$

Nonadherence to treatments for chronic therapies has long been recognized as a major contributor to excess hospitalizations and wasteful health care spending. ${ }^{18-25}$ For patients with immune-mediated diseases, who now comprise $5 \%-7 \%$ of the Western population, studies have shown that better medication-taking behavior is associated with lower risk of hospital visits, including inpatient admissions and emergency department (ED) visits, which account for the majority of disease-related medical costs. ${ }^{1,4,7-11}$ Common barriers to adherence for immune-mediated disease patients include intentional (e.g., fear of adverse events, efficacy concerns, financial constraints, difficulty navigating insurance) and unintentional (e.g., forgetfulness, difficulty with refills) factors. ${ }^{26-28}$ To address such hurdles, PSPs provide individualized counseling, training, and assistance to motivate patients and improve medication-taking behavior, but the sustainability of these benefits and their impact on hospital visits is unknown. ${ }^{3,22,28}$

Since 2015, AbbVie Inc. (North Chicago, IL) has offered a unique PSP for adalimumab patients, HUMIRA Complete, designed to provide one-on-one, personalized support to increase medication adherence through behavior modification and motivate patients to improve their health literacy. The program offers assistance with accessing treatment (including education on financial assistance options and insurance coverage requirements), emotional motivation, treatment management, disease education, medication reminders, and a dedicated Nurse Ambassador (a registered nurse trained to help patients adhere to their prescribed treatment plan but who does not provide clinical guidance). Prior studies demonstrated the impact of this PSP in improving access to treatment, medication-taking behavior, and costs, but studies were limited to the first 12 months of adalimumab treatment and did not examine hospital visits. ${ }^{1,2,13,14}$ Sufficient data are now available to provide more complete evidence on the benefits of PSPS throughout the duration of treatment by examining the impact of PSP use on sustained medication-taking behavior and risk of hospital visits.

The research presented here is an extension study to assess if improvements in medication-taking behavior observed for PSP participants continued beyond the first 12 months of treatment and to evaluate occurrences of hospital visits. ${ }^{1}$ Up to 36 months of longitudinal, patient-level data are now available, providing an opportunity to evaluate 
the sustainability of PSP benefits and the potential to lower the risk of clinically important and costly hospital visits for patients with immune-mediated diseases.

\section{Methods}

\section{STUDY DESIGN AND DATA SOURCES}

A retrospective, longitudinal matched-cohort study was conducted using patient-level data from the Symphony Health administrative claims database and PSP enrollment records, details of which have been described previously. For this study, claims were available from January 2006 through April 2019. A proprietary deidentification engine (Synoma, Symphony Health, Phoenix, AZ) was used by each data provider to generate unique patient tokens based on identifiable information that was removed from the data provided to researchers, allowing for direct patient linking between the PSP records and claims. Any risk associated with linked data content was evaluated by an external HIPAA statistician who certified patient anonymity of the resulting files. Because deidentification was conducted before providing claims to Symphony Health and PSP records to researchers, and no identifiable protected health information was included in the data used, institutional review board approval was not required. As this was an extension study of a preexisting patient cohort, it was not registered publicly prior to conducting the analysis.

\section{STUDY POPULATION}

Eligibility Criteria. The methodology used to create the study sample has been previously described. ${ }^{1}$ Patients initiating adalimumab were included if they were at least 18 years of age and had an adalimumab indication (rheumatoid arthritis [RA], Crohn's disease [CD], ulcerative colitis [UC], psoriasis, psoriatic arthritis [PsA], ankylosing spondylitis [AS], uveitis, and hidradenitis suppurativa [HS]), were biologic-naive prior to adalimumab initiation, and did not have government-provided insurance on their initial adalimumab claim, which would make them ineligible for the financial assistance component of the PSP. The index date was defined as the date of PSP enrollment for PSP patients. The length of time between the initial adalimumab claim and PSP enrollment for PSP patients was used to calculate the index date for matched controls as described below.

Claims Criteria. Each patient was required to have $\geq 2$ claims at least 30 days apart with International Classification of Diseases, Ninth/Tenth Revision, Clinical Modification (ICD9/10-CM) diagnosis codes for an adalimumab indication (Supplementary Table 1, available in online article). The first adalimumab claim must have occurred between January
2015 and February 2016 and evidence of continuous medical and drug data coverage was required for at least 6 months before and at least 12 months after the first adalimumab claim and index date (if different). Periods of continuous data coverage were determined based on the observed frequency of medical and drug claims using previously developed algorithms. ${ }^{1,2}$ The 6 -month period before the index date was considered the baseline period. Follow-up period outcomes were evaluated at 12, 24, and 36 months after the index date for patients with sufficient data availability.

Cohort Assignment and Matching Process. Patients who met the eligibility criteria were categorized into 2 cohorts, the PSP cohort and the non-PSP cohort, based on enrollment in the PSP within 30 days of adalimumab initiation and engagement with a Nurse Ambassador. PSP patients were matched 1:1 to non-PSP patients based on pharmacy type of the initial adalimumab claim, immune-mediated disease indication, and propensity score estimated with covariates for age, sex, year of adalimumab initiation, and baseline comorbidities. Details of the cohort construction and rationale for the matching process used have been previously described. ${ }^{1}$

\section{OUTCOMES}

Measures of medication-taking behavior (adherence and persistence) and hospital visits were assessed at 12, 24, and 36 months following the index date. Adherence was measured using the proportion of days covered (PDC): the sum of the number of days covered by the reported days supply of adalimumab claims divided by the number of days in each follow-up period (e.g., 365 days for 12-month PDC). Persistence was defined as the proportion of patients remaining on treatment throughout each follow-up period, with discontinuation determined by a switch to another biologic medication or a gap in adalimumab treatment exceeding the days supply of the prior adalimumab claim with no further adalimumab claim during the follow-up period. Duration of persistence was calculated as the time from the index date to the earlier of discontinuation or the end of data availability. Hospital visits, including inpatient admissions and ED visits, were identified based on the location of service reported on medical claims, and the proportion of patients with at least 1 hospital visit was summarized at each timepoint. Time to a hospital visit was calculated as the time elapsed from the index date to the first occurrence of a hospital visit.

\section{STATISTICAL ANALYSES}

Baseline demographics, clinical characteristics, and hospital visits for the 6 months prior to the index date were 


\section{FIGURE 1 Adherence to Adalimumab at 12, 24, and 36 Months $^{\mathrm{a}, \mathrm{b}}$}

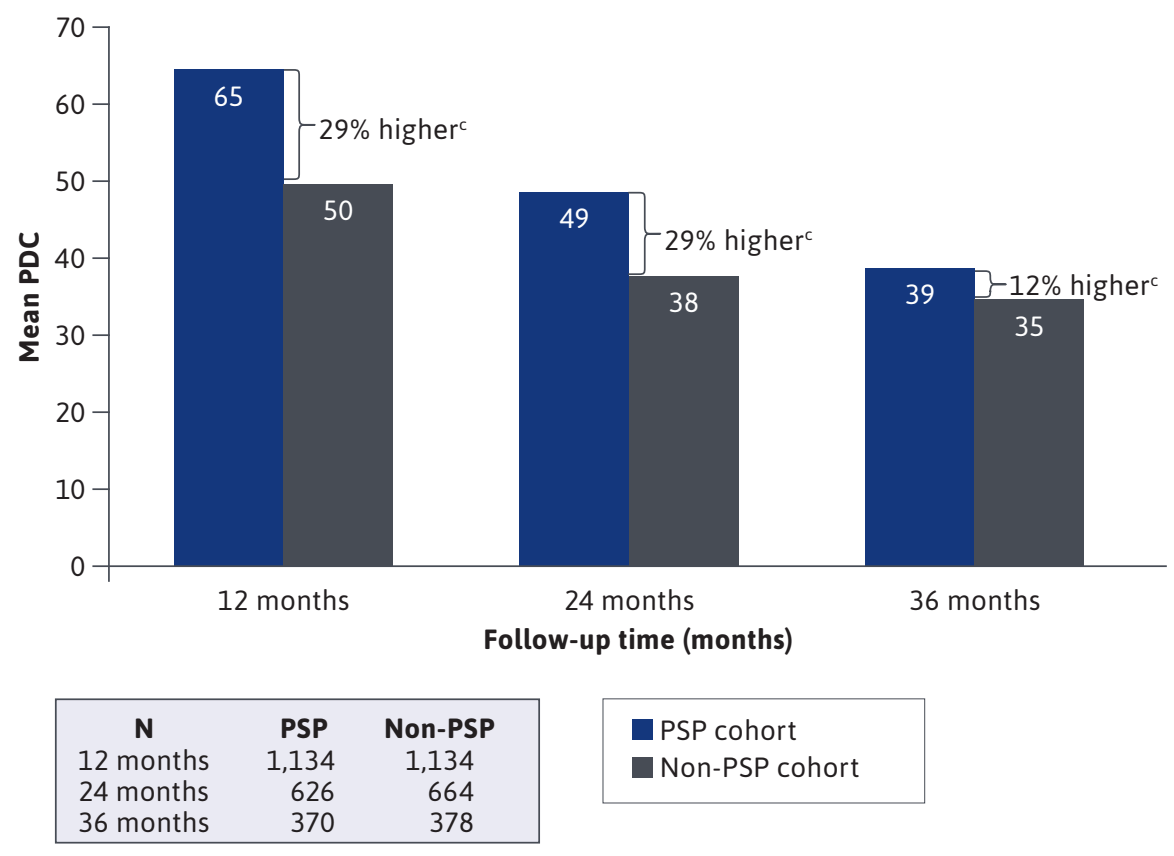

aRelative differences calculated as (PSP - non-PSP)/(non-PSP).

${ }^{b} P$ values adjusted for baseline characteristics and hospital visits.

${ }^{c} P<0.05$.

$P D C=$ proportion of days covered; $P S P=$ patient support program

summarized using descriptive statistics. Differences in baseline measures and follow-up data availability between cohorts were assessed using Student's t-tests for continuous variables and chi-square tests for categorical variables. Outcomes were descriptively evaluated using means, medians, proportions, standard deviations (SDs), and 95\% confidence intervals (CIs) where appropriate. Relative differences were calculated as the difference between the estimates for the PSP and non-PSP cohorts divided by the estimate for the nonPSP cohort. Separate multivariable generalized linear models were used to compare outcomes across cohorts at 12,24 , and 36 months, and hazard ratios (HRs) of the effect of PSP participation on discontinuation and hospital visits were estimated using Cox proportional-hazards models. All models controlled for baseline demographics, clinical characteristics, and hospital visits. Duration of persistence and time to a hospital visit were compared between cohorts using Kaplan-Meier analyses, censoring for loss of patient-level follow-up data. Outcomes were estimated for the full sample and separately for each therapeutic area (rheumatology [RA, PsA, and AS], gastroenterology [CD and $\mathrm{UC}$ ], and dermatology [psoriasis and HS]; uveitis did not have sufficient sample size to analyze individually). All analyses were conducted using SAS software version 9.4 (SAS Institute, Cary, NC).

\section{Results}

\section{SAMPLE AND DATA AVAILABILITY}

The matched sample included 2,268 patients (1,134 in each cohort; Supplementary Figure 1, available in online article), with similar baseline characteristics and hospital visits (Supplementary Table 2, available in online article). As reported previously, the mean age of the study population was 50.4 years, and approximately $70 \%$ of the patient population was female. The most common indications for adalimumab treatment in both cohorts were RA (41.4\% of patients), CD (19.5\%), psoriasis (14.3\%), and UC (13.2\%). No significant difference was found in the proportion of patients with a hospital visit during the baseline period (20.6\% for PSP vs. 23.5\% for non-PSP; $\mathrm{P}=0.09)$. Longitudinal, patient-level follow-up data availability was also similar across cohorts (mean [SD] duration was 26.2 [8.7] months for the PSP cohort vs. 26.5 [8.7] months for the non-PSP cohort; $P=0.36$ ). Patients in the PSP cohort remained engaged with the program for 16.8 months on average, while $12 \%$ of the non-PSP cohort opted into the PSP during the follow-up period (after not enrolling when initiating treatment).

\section{MEDICATION-TAKING BEHAVIOR}

Medication-taking behavior, as assessed by adherence and persistence, was higher for the PSP cohort at each follow-up timepoint. The PSP cohort consistently demonstrated higher adalimumab adherence than the non-PSP cohort at 12 (64.8\% vs. 50.1\%, $\mathrm{P}<0.0001 ; 29 \%$ greater), 24 (49.4\% vs. $38.4 \% ; \mathrm{P}<0.0001 ; 29 \%$ greater), and 36 (39.4\% vs. $35.1 \% ; \mathrm{P}=0.02 ; 12 \%$ greater) months (Figure 1). Similarly, a greater proportion of PSP patients were persistent on adalimumab at 12 (49\% vs. $34 \%$; $\mathrm{P}<0.0001 ; 42 \%$ greater), 24 (36\% vs. $26 \% ; \mathrm{P}<0.0001 ; 37 \%$ greater), and 36 


\section{FIGURE 2 Adalimumab Persistence and Discontinuation over 36 Months $s^{\mathrm{a}, \mathrm{b}}$}

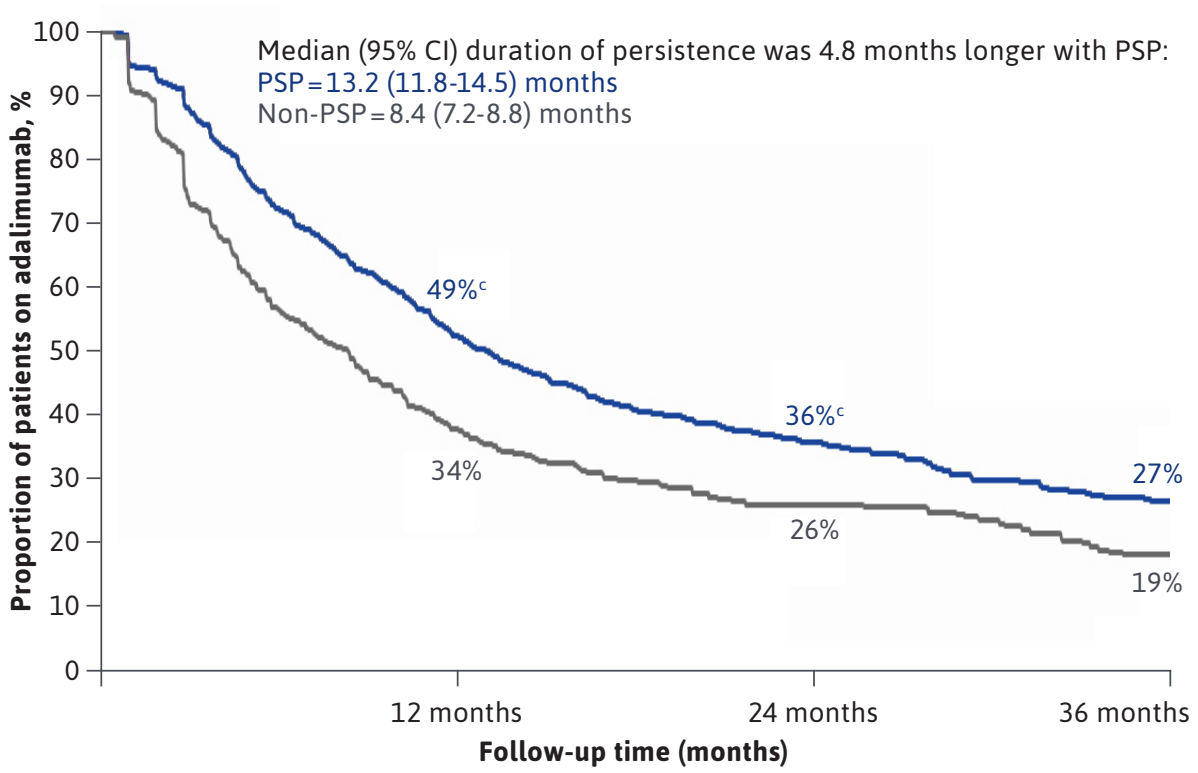

Risk of discontinuation was $30 \%$ lower with PSP vs. non-PSP ( $\mathrm{HR}=0.70,95 \% \mathrm{Cl}=0.64-0.78 ; P<0.0001)$

PSP cohort $(\mathrm{N}=1,134) \quad$ Non-PSP cohort $(\mathrm{N}=1,134)$

a Median duration of persistence calculated from Kaplan-Meier survival analysis.

${ }^{b} P$ values and HR estimates adjusted for baseline characteristics and hospital visits.

${ }^{c} P<0.05$.

$\mathrm{Cl}=$ confidence interval; $\mathrm{HR}=$ hazard ratio; $\mathrm{PSP}=$ patient support program

(27\% vs. 19\%; $\mathrm{P}<0.0001 ; 44 \%$ greater) months (Figure 2). The adjusted risk of adalimumab discontinuation was $30 \%$ lower for the PSP cohort relative to the non-PSP cohort $(\mathrm{HR}=0.70$ $[95 \% \mathrm{CI}=0.64-0.78] ; \mathrm{P}<0.0001)$. Median duration of persistence with adalimumab was 4.8 months longer for the PSP cohort than for the non-PSP cohort (13.2 [95\% CI=11.8-14.5] months vs. $8.4[95 \% \mathrm{CI}=7.2-8.8]$ months; $\log$-rank $\mathrm{P}<0.0001)$. Improved medication-taking behavior for the PSP cohort, including higher adherence, lower risk of discontinuation, and longer duration of persistence, was found across all therapeutic areas
(Supplementary Table 3, available in online article).

\section{HOSPITAL VISITS}

The PSP cohort had lower rates of hospital visits at each follow-up timepoint. The PSP cohort had fewer patients with a hospital visit at 12 (30\% vs. 37\%; $\mathrm{P}<0.001 ; 19 \%$ lower), 24 (44\% vs. 53\%; $\mathrm{P}=0.01 ; 17 \%$ lower), and 36 (55\% vs. 65\%; $\mathrm{P}<0.01 ; 16 \%$ lower) months (Figure 3). In survival analyses, time to a hospital visit was significantly longer for the PSP cohort (log-rank $\mathrm{P}<0.0001)$, with the median time to a hospital visit of 11.8 months longer than for the non-PSP cohort
(32.7 [95\% CI=29.1-NA] vs. 21.9 [95\% $\mathrm{CI}=18.7-24.9]$; Figure 4). After adjusting for baseline characteristics, the PSP cohort had a $25 \%$ lower risk of a hospital visit $(\mathrm{HR}=0.75$ [95\% $\mathrm{CI}=0.66$ $0.85]$; $\mathrm{P}<0.0001)$ over 36 months.

Findings were similar for ED visits and inpatient admissions examined separately. The PSP cohort had fewer patients with an ED visit (13\%-24\% lower) or inpatient admission (16\%$22 \%$ lower) at each follow-up period (all $\mathrm{P}<0.05$, except 12-month inpatient admissions; Figure 3). After adjusting for baseline characteristics, the PSP cohort had a $28 \%$ lower risk of an $\mathrm{ED}$ visit $(\mathrm{HR}=0.72$ [95\% $\mathrm{CI}=0.63-0.83]$; $\mathrm{P}<0.0001)$ and a $17 \%$ lower risk of inpatient admission $(\mathrm{HR}=0.83 \quad[95 \%$ $\mathrm{CI}=0.71-0.97] ; \quad \mathrm{P}=0.0220) \quad$ over 36 months (Figure 4).

Results were also consistent across therapeutic areas, with greater improvements in medication-taking behavior corresponding with lower risk of hospital visits (Supplementary Table 3, available in online article). After adjusting for baseline characteristics, the PSP cohort had a $28 \%$, $27 \%$, and $37 \%$ lower risk of a hospital visit for rheumatology $(\mathrm{HR}=0.72$ [95\% $\mathrm{CI}=0.60-0.87]), \quad$ gastroenterology $(\mathrm{HR}=0.73 \quad[95 \% \mathrm{CI}=0.60-0.89])$, and dermatology $(\mathrm{HR}=0.63[95 \% \mathrm{CI}=0.43-$ 0.91]) patients participating in the PSP (all $\mathrm{P}<0.05$ ).

\section{Discussion}

The research presented here provides novel evidence of the association between PSP participation, long-term medication-taking behavior, and hospital visits, an important driver of health care costs among patients with immune-mediated diseases. Leveraging an innovative data-linking initiative conducted to support a prior evaluation of early outcomes for PSP patients, the combination of longitudinal, real-world claims with PSP 


\section{FIGURE 3 Hospital Visits at 12, 24, and 36 Months $^{\mathrm{a}-\mathrm{c}}$}

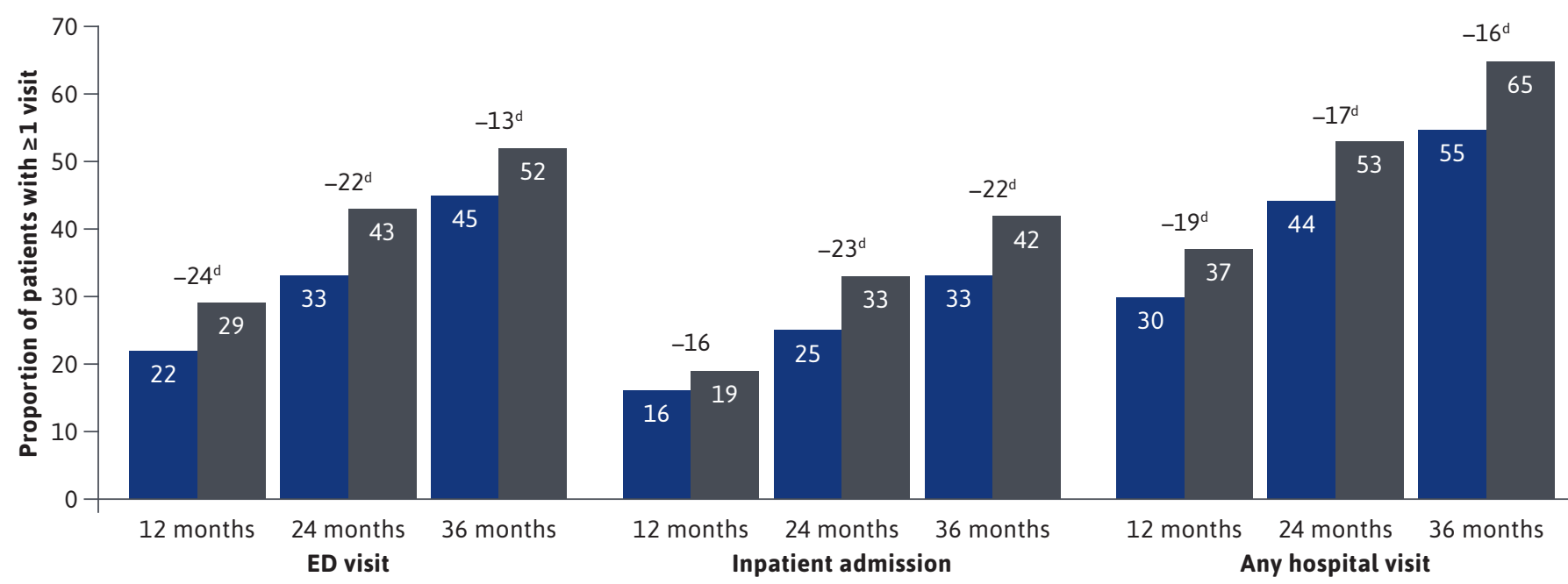

\begin{tabular}{|crc|}
\hline $\mathbf{N}$ & PSP & Non-PSP \\
12 months & 1,134 & 1,134 \\
24 months & 626 & 664 \\
36 months & 370 & 378 \\
\hline
\end{tabular}

${ }^{a}$ Relative differences calculated as (PSP - non-PSP)/(non-PSP).

${ }^{b} P$ values adjusted for baseline characteristics and hospital visits.

'Any hospital visit is a composite measure of either an ED visit or inpatient admission.

${ }^{d} P<0.05$.

$E D=$ emergency department; $P S P=$ patient support program.

utilization records allowed for examination of long-term treatment outcomes and rare hospital visit events. Over 36 months of follow-up, adalimumab patients who participated in AbbVie's PSP sustained higher levels of adherence and persistence and a lower risk of a hospital visit than patients who did not participate. These findings inform the potential effectiveness and benefits of patient-centric initiatives to improve outcomes and lower the burden to the health care system.

Hospital care comprises a third of all health care spending in the United States, with the growth rate in the cost of hospital care outpacing all other medical services in recent decades - a trend that is projected to continue for the foreseeable future. ${ }^{29-31}$ Approximately $10 \%$ of hospitalizations for patients with chronic diseases (and 33\%-69\% of medication-related hospital admissions) have been attributed to suboptimal adherence, and hospitalizations account for the majority of estimated costs of nonadherence, a leading contributor to avoidable medical spending. ${ }^{21-25,32,33}$ Extrapolating the hospital visit results presented here using national average costs of ED visits and inpatient admissions, PSP participation could have reduced hospital care costs by $\$ 1.7$ million for the non-PSP cohort, or $\$ 438$ million for all adalimumab patients in the United States over 36 months $-22 \%$ of total hospital care costs for adalimumab patients. $^{34}$

While improved medication-taking behavior is crucial in minimizing disease flares and reducing hospital visits, other components of the PSP may provide additional benefits for patients utilizing the program. ${ }^{7,8,10}$ Nurse Ambassadors, for example, provide education, cognitive support, and motivation for patients to improve health literacy of their disease and treatment. Prior studies have hypothesized that such high-touch and individualized initiatives incorporating multiple support mechanisms to address specific challenges facing patients with chronic diseases are likely to substantially improve a wide range of outcomes, including but not limited to hospital visits. ${ }^{3,12,23,28,35-39}$ Other direct benefits could include lower rates of outpatient health care resource use, prescriptions for comorbid conditions, diagnostic 


\section{FIGURE 4 Risk of Hospital Visits over 36 Months $^{\mathrm{a}-\mathrm{c}}$}

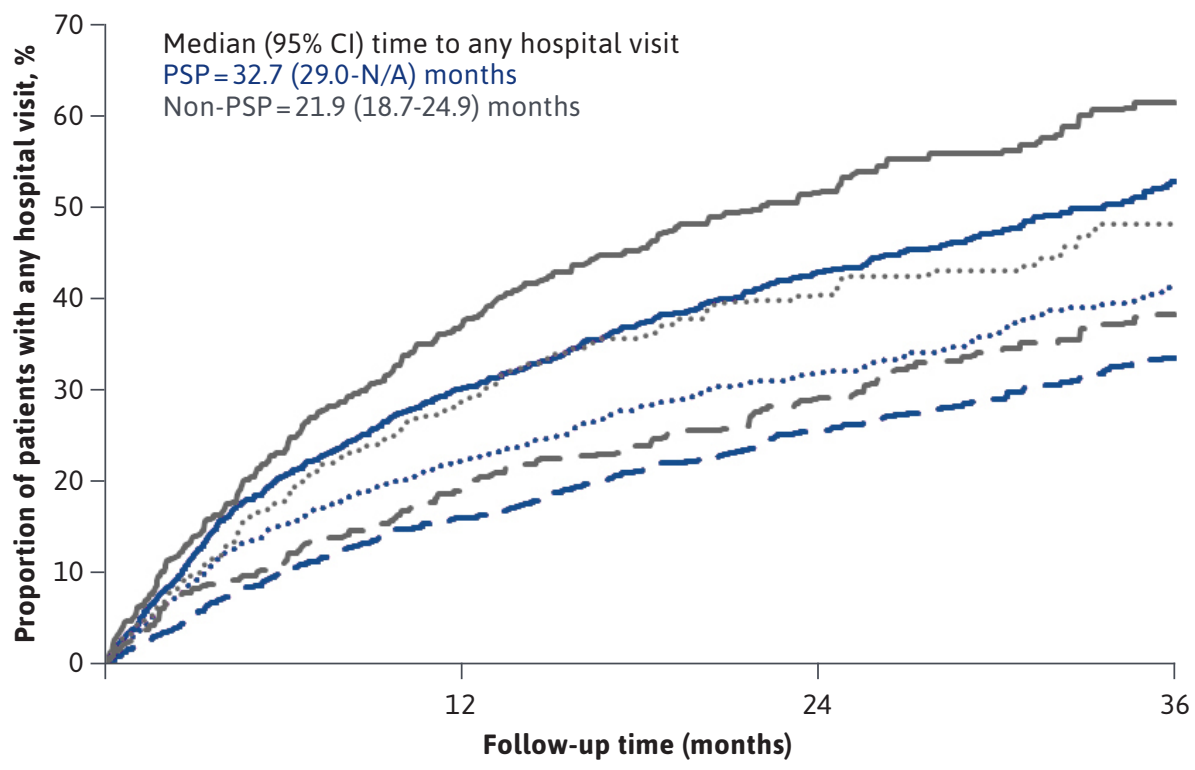

PSP patients had lower risk of hospital visits than non-PSP patients:

- $28 \%$ lower risk of $\mathrm{ED}$ visit $(\mathrm{HR}=0.72 ; 95 \% \mathrm{Cl}=0.63-0.83 ; P<0.0001)$

- $17 \%$ lower risk of inpatient admission $(\mathrm{HR}=0.83 ; 95 \% \mathrm{Cl}=0.71-0.97 ; \mathrm{P}=0.0220)$

- $25 \%$ lower risk of any hospital visit $(\mathrm{HR}=0.75 ; 95 \% \mathrm{Cl}=0.66-0.85 ; P<0.0001)$

$$
\begin{array}{ll}
\text { — PSP any hospital visit } & \text { - Non-PSP any hospital visit } \\
\text {...... PSP ED visit } & \ldots . . \text { Non-PSP ED visit } \\
\text { - - PSP inpatient admission } & - \text { - Non-PSP inpatient admission }
\end{array}
$$

Note: Log-rank $P<0.05$ in each comparison.

${ }^{a}$ Failure curves estimated using Kaplan-Meier analysis censoring for loss of data.

${ }^{b} \mathrm{HR}$ estimates from Cox-proportional hazard models adjusted for baseline characteristics and hospital visits.

'Any hospital visit is a composite measure of either an ED visit or inpatient admission.

$E D=$ emergency department; $\mathrm{Cl}=$ confidence interval; $H R=$ hazard ratio; $P S P=$ patient support program.

testing, and surgical interventions, while indirect benefits could include reduced physical suffering and better quality of life. ${ }^{22,23,40}$ However, evidence has typically been restricted to shortterm outcomes; only 1 of 58 studies included in a recent systematic review of self-management support strategies followed patients beyond 12 months (none in immune-mediated diseases), and systematic reviews of electronic interventions to educate patients and improve adherence overall and in chronic diseases specifically did not find any studies longer than 12 months (or in immune-mediated diseases). ${ }^{12,41,42}$ The sustainability of benefits is often unknown but critical to the effectiveness of support interventions. ${ }^{12,43}$ This study provides the first evidence that early improvements for immune-mediated disease patients participating in the PSP continue up to 36 months following treatment initiation.
Long-term improvements in medication-taking behavior and lower risk of hospitalization were found across all therapeutic areas. Dermatology patients utilizing the PSP experienced the greatest relative reduction in hazard of both discontinuation and a hospital visit, though significant decreases were also seen in rheumatology and gastroenterology. The reductions found among gastroenterology patients, while relatively smaller in magnitude than in dermatology, may be the most consequential given the higher rate of hospitalizations for gastroenterology patients, which contribute to the high (and increasing) costs of the disease. ${ }^{44}$ Providing validation, the impact of PSP participation on the risk of hospital visits for gastroenterology patients found in the current study is consistent with a recent 12-month analysis of the same PSP using a closed-claims database. ${ }^{45}$ Further research should continue to examine heterogeneity in the benefits of PSPs across therapeutic areas to better understand their potential to lower the risk of hospital visits for the broader chronic disease population.

The intent of this study was to examine real-world outcomes for patients enrolling in the PSP at the time of biologic initiation, and thus findings on the benefits of PSP use are expected to be conservative, as patients in the PSP cohort were not required to remain on adalimumab (which would have increased the medication-taking behavior estimates) or to have remained actively engaged with the PSP (though mean participation was 17 months) during the follow-up period. Conversely, patients in the non-PSP cohort were allowed to enroll in the PSP during the follow-up period (which $12 \%$ of the sample did). Both dynamics are expected to make the observed differences in medication-taking behavior and hospital visits conservative. Improvements in 
adherence and persistence that were previously observed during the first 12 months of treatment with the PSP were sustained up to 24 months, before tapering in magnitude slightly at 36 months (though both adherence and persistence remained higher than for the non-PSP cohort). The decreased magnitude of adherence differences over time (from 29\% to 12\% higher for the PSP cohort relative to the non-PSP cohort) is expected as patients discontinue adalimumab therapy. Similarly, relative reductions in hospital visits at 36 months were slightly lower than observed at 24 months, likely due to discontinuation of adalimumab and/ or PSP participation. Though not presented, hospital visit rates were lowest for patients who remained persistent on adalimumab therapy and actively engaged with the PSP throughout the follow-up period.

The importance of treatment adherence to clinical, economic, and societal outcomes has long been recognized, which makes the observed low rates of adherence to biologic therapies for immune-mediated diseases a cause for concern. Initiatives to improve adherence present an opportunity to reduce avoidable health care resource use and curb increases in medical spending. As more attention has been paid to such efforts, it has become clear that effective initiatives provide multiple components of personalized support that are maintained throughout the course of treatment. This study of one such PSP illustrates the long-term medication-taking behavior benefits, along with the lower risk of hospital visits, across several therapeutic areas. The results provide further evidence that PSPs should be considered a potentially valuable service for patients prescribed biologic therapies for immunemediated diseases.

\section{LIMITATIONS}

As with all retrospective studies, limitations of the data sources and study design should be noted, many of which have been previously reported. ${ }^{1}$ Notably, the evaluation of hospital visits required medical claims; however, any visits to providers not captured by Symphony Health would not be observed. While this may downwardly bias hospital visit estimates, there is no reason to expect that the relative difference between cohorts would be affected. The evaluation of medication-taking behavior depended on pharmacy claims, which may not reflect actual administration of the drug, and reasons for nonadherence or discontinuation (e.g., physician direction or patient volition) could not be determined. As such, comparisons of adherence and discontinuation focused on the relative differences across cohorts, which are less sensitive to potential sources of bias (e.g., missing claims) than the absolute levels.
An important limitation of any cohort study is the potential for selection bias (i.e., patients enrolling in the PSP may differ from those not enrolling on characteristics not observable in the data but related to the outcomes assessed). To address this concern, patients were matched on an extensive list of covariates, which were also adjusted for in the estimation of outcomes; however, unobserved heterogeneity could remain.

When the cohorts were constructed, available follow-up data beyond 12 months was not known, and thus patient attrition could vary. Though follow-up length did not differ significantly across cohorts and all analyses were censored for loss of follow-up to mitigate this concern, if data availability was correlated with cohort selection or the outcomes assessed, bias could remain. However, given that results were consistent when comparisons were conducted only among patients with sufficient follow-up data, it does not seem patient attrition bias significantly affected any of the findings regarding the benefits of PSP participation.

Patients participating in the PSP used several components simultaneously, so the incremental effect of any particular component, such as injection training or education on financial assistance options, could not be evaluated. Missing from this evaluation of PSP participation is an assessment of patient satisfaction, including which services improved medication-taking behavior and what barriers remained, which should be included in future research.

Finally, this study included commercially insured patients initiating adalimumab for immune-mediated diseases, and results may not be generalizable to other patient populations, treatments, or PSPs.

\section{Conclusions}

This study provides novel evidence that participation in a comprehensive, individualized PSP was associated with sustained, long-term improvements in medication-taking behavior and hospital visits for patients initiating adalimumab therapy. Over 36 months, patients who participated in the PSP had higher levels of adherence and persistence and lower risk of hospital visits, including inpatient admissions and ED visits, than patients who did not participate. These findings build on short-term evidence that PSP participation improves patient outcomes and lowers the burden to the health care system. Further research should examine specific aspects of the PSP associated with improved outcomes to inform the design of patient-centric initiatives in other patient populations. 


\section{DISCLOSURES}

Design, study conduct, and financial support for the study were provided by AbbVie Inc., which participated in the interpretation of data, review, and approval of the manuscript.

Fendrick has received personal fees from Merck, AstraZeneca, Trizetto, Amgen, Lilly, AbbVie, Johnson \& Johnson, and Sanofi; grants from the National Pharmaceutical Council, PhRMA, the Gary and Mary West Health Foundation, the states of New York and Michigan, the Laura and John Arnold Foundation, the Robert Wood Johnson Foundation, and the Agency for Healthcare Research and Quality; and equity in Zansors, Sempre Health, Wellth, and V-BID Health.

Brixner has received consulting fees from AbbVie, Novartis, Xcenda, Elevar Therapeutics, Sanofi, UCB Pharma, and the Millcreek Outcomes Group.

Rubin has received consulting fees from AbbVie, Abgenomics, Allergan Inc., Amgen, Celgene Corporation, Forward Pharma, Genentech/Roche, Janssen Pharmaceuticals, Merck \& Co., Miraca Life Sciences, Mitsubishi Tanabe Pharma Development America, Napo Pharmaceuticals, Pfizer, Salix Pharmaceuticals Inc., Samsung Bioepis, Sandoz Pharmaceuticals, Shire, Takeda, and Target Pharmaceuticals; and research support from AbbVie, Genentech/Roche, Janssen Pharmaceuticals, Prometheus Laboratories, Shire, Takeda, and UCB Pharma.

Mease has received grant/research support from AbbVie, Amgen, BMS, Celgene, Janssen, Lilly, Merck, Novartis, Pfizer, SUN Pharma, and UCB; consulting fees from AbbVie, Amgen, BMS, Boehringer Ingelheim, Celgene, Galapagos, Genentech, Gilead, GlaxoSmithKline, Janssen, Lilly, Novartis, Pfizer, SUN Pharma, and UCB; and has served on the speakers bureau for AbbVie, Amgen, Celgene, Janssen, Lilly, Novartis, Pfizer, and UCB.

Liu has no financial conflict of interest. Davis is an employee of Medicus Economics, which received payment from AbbVie to participate in this research. Mittal is an employee and stockholder of AbbVie.

This study used a cohort of patients previously described in Brixner D, Rubin DT, Mease P, et al. Patient support program increased medication adherence with lower total health care costs despite increased drug spending. J Manag Care
Spec Pharm. 2019 Jul;25(7):770-79 (doi: 10.18553/jmcp.2019.18443). As such, the sample selection and select baseline characteristics and 12-month outcomes have been published previously; however, the hospital visit outcomes and the longer-term medication-taking behavior outcomes have not been previously published or presented.

\section{ACKNOWLEDGMENTS}

Medical writing support was provided by Taylor Renteria of Medicus Economics and funded by AbbVie.

\section{REFERENCES}

1. Brixner D, Rubin DT, Mease P, et al. Patient support program increased medication adherence with lower total health care costs despite increased drug spending. J Manag Care Spec Pharm. 2019;25(7):770-79. doi:10.18553/ jmcp.2019.18443

2. Rubin DT, Mittal M, Davis M, Johnson S, Chao J, Skup M. Impact of a patient support program on patient adherence to adalimumab and direct medical costs in Crohn's disease, ulcerative colitis, rheumatoid arthritis, psoriasis, psoriatic arthritis, and ankylosing spondylitis. J Manag Care Spec Pharm. 2017;23(8) 859-67. doi:10.18553/jmcp.2017.16272.

3. Ganguli A, Clewell J, Shillington AC. The impact of patient support programs on adherence, clinical, humanistic, and economic patient outcomes: a targeted systematic review. Patient Prefer Adherence. 2016;10:711-25.

4. Maniadakis N, Toth E, Schiff M, et al. A targeted literature review examining biologic therapy compliance and persistence in chronic inflammatory diseases to identify the associated unmet needs, driving factors, and consequences. Adv Ther. 2018;35(9):1333-55.

5. Murage MJ, Tongbram V, Feldman SR, et al. Medication adherence and persistence in patients with rheumatoid arthritis, psoriasis, and psoriatic arthritis: a systematic literature review. Patient Prefer Adherence. 2018;12:1483-1503.
6. Bluett J, Morgan C, Thurston L, et al. Impact of inadequate adherence on response to subcutaneously administered anti-tumour necrosis factor drugs: results from the Biologics in Rheumatoid Arthritis Genetics and Genomics Study Syndicate cohort. Rheumatology (Oxford). 2015;54(3):494-99.

7. Gendelman O, Weitzman D, Rosenberg V, Shalev V, Chodick G, Amital H.

Characterization of adherence and persistence profile in a real-life population of patients treated with adalimumab. $\mathrm{Br} \mathrm{J}$ Clin Pharmacol. 2018;84(4):786-95.

8. Govani SM, Noureldin M, Higgins PDR, et al. Defining an optimal adherence threshold for patients taking subcutaneous anti-TNFs for inflammatory bowel diseases. Am J Gastroenterol. 2018;113(2):276-82.

9. Kane SV, Chao J, Mulani PM. Adherence to infliximab maintenance therapy and health care utilization and costs by Crohn's disease patients. Adv Ther. 2009;26(10):936-46.

10. Lathia U, Ewara EM, Nantel F. Impact of adherence to biological agents on health care resource utilization for patients over the age of 65 years with rheumatoid arthritis. Patient Prefer Adherence. 2017;11:1133-42.

11. Carter CT, Waters HC, Smith DB. Impact of infliximab adherence on Crohn's disease-related healthcare utilization and inpatient costs. Adv Ther. 2011;28(8):671-83

12. Dineen-Griffin S, Garcia-Cardenas V, Williams K, Benrimoj SI. Helping patients help themselves: a systematic review of self-management support strategies in primary health care practice. PLoS One. 2019;14(8):e0220116.

13. Brixner D, Mittal M, Rubin DT, et al Participation in an innovative patient support program reduces prescription abandonment for adalimumab-treated patients in a commercial population. Patient Prefer Adherence. 2019;13:1545-56.

14. Hawkes JE, Mittal M, Davis M, Brixner D. Impact of online prescription management systems on biologic treatment initiation. Adv Ther. 2019;36(8):2021-33. 
15. El-Gabalawy H, Guenther LC, Bernstein CN. Epidemiology of immunemediated inflammatory diseases: incidence, prevalence, natural history, and comorbidities. J Rheumatol Suppl. 2010;85:2-10.

16. Jacobs P, Bissonnette R, Guenther LC. Socioeconomic burden of immune-mediated inflammatory diseases-focusing on work productivity and disability.

J Rheumatol Suppl. 2011;88:55-61.

17. Ford AC, Sandborn WJ, Khan KJ, et al. Efficacy of biological therapies in inflammatory bowel disease: systematic review and meta-analysis. Am J Gastroenterol. 2011;106(4):644-59.

18. Berwick DM, Hackbarth AD. Eliminating waste in U.S. health care. JAMA. 2012;307(14):1513-16.

19. Shrank WH, Rogstad TL, Parekh N. Waste in the U.S. health care system: estimated costs and potential for savings. JAMA. 2019;322(15):1501-09.

20. Roebuck MC, Liberman JN, Gemmil-Toyama M, Brennan T. Medication adherence leads to lower health care use and costs despite increased drug spending. Health Aff (Millwood). 2011;30(1):91-99.

21. New England Healthcare Institute. Thinking outside the pillbox: a systemwide approach to improving patient medication adherence for chronic disease. NEHI Research Brief. 2009. Accessed March 21, 2021. https://www.nehi.net/ writable/publication files/file/pa issue_brief_final.pdf

22. Iuga AO, McGuire MJ. Adherence and health care costs. Risk Manag Healthc Policy. 2014;7:35-44.

23. Martin LR, Feig C, Maksoudian CR, Wysong K, Faasse K. A perspective on nonadherence to drug therapy: psychological barriers and strategies to overcome nonadherence. Patient Prefer Adherence. 2018;12:1527-35.

24. Cutler RL, Fernandez-Llimos F, Frommer M, et al. Economic impact of medication non-adherence by disease groups: a systematic review. BMJ Open. 2018;8(1):e016982.

25. Watanabe JH, McInnis T, Hirsch JD. Cost of prescription drug-related morbidity and mortality. Ann Pharmacother. 2018;52(9):829-37.
26. Wohlfahrt A, Campos A, Iverson MD et al. Use of rheumatology-specific patient navigators to understand and reduce barriers to medication adherence: analysis of qualitative findings. PLoS One. 2018;13(7):e0200886.

27. Betegnie AL, Gauchet A, Lehmann A, et al. Why do patients with chronic inflammatory rheumatic diseases discontinue their biologics? An assessment of patients' adherence using a self-report questionnaire. J Rheumatol. 2016;43(4):724-30

28. Demoly P, Passalacqua G, Pfaar O, Sastre J, Wahn U. Patient engagement and patient support programs in allergy immunotherapy: a call to action for improving long-term adherence. Allergy Asthma Clin Immunol. 2016;12:34.

29. Centers for Medicare \& Medicaid Services. National Health expenditures 2019 highlights. Accessed March 31, 2021. https://www.cms.gov/files/document/ highlights.pdf

30. Keehan SP, Cuckler GA, Poisal JA, et al. National health expenditure projections, 2019-28: expected rebound in prices drives rising spending growth. Health Aff (Millwood). 2020;39(4):704-14.

31. Cooper Z, Craig S, Gaynor M, et al. Hospital prices grew substantially faster than physician prices for hospital-based care in 2007-14. Health Aff (Millwood). 2019;38(2):184-89.

32. Osterberg L, Blaschke T. Adherence to medication. N Engl J Med. 2005;353(5):487-97.

33. Sokol MC, McGuigan KA, Verbrugge RR, Epstein RS. Impact of medication adherence on hospitalization risk and healthcare cost. Med Care. 2005;43(6):521-30.

34. Agency for Healthcare Research and Quality. Medication Expenditure Panel Survey. 2018. Accessed March 31, 2021. https://www.meps.ahrq.gov/mepsweb/

35. Hardman R, Begg S, Spelten E. What impact do chronic disease self-management support interventions have on health inequity gaps related to socioeconomic status: a systematic review. BMC Health Serv Res. 2020;20(1):150.
36. Allegrante JP, Wells MT, Peterson JC. Interventions to support behavioral selfmanagement of chronic diseases. Annu Rev Public Health. 2019;40:127-46.

37. Sapkota S, Brien J, Greenfield J, Aslani P. A systematic review of interventions addressing adherence to anti-diabetic medications in patients with type 2 diabetes-impact on adherence. PLoS One. 2015;10(2):e0118296.

38. Nieuwlaat R, Wilczynski N, Navarra T, et al. Interventions for enhancing medication adherence. Cochrane Database Syst Rev. 2014;2014(11):Cd000011.

39. Viswanathan M, Golin CE, Jones CD, et al. Interventions to improve adherence to self-administered medications for chronic diseases in the United States: a systematic review. Ann Intern Med. 2012;157(11):785-95.

40. Martin LR, Williams SL, Haskard KB, Dimatteo MR. The challenge of patient adherence. Ther Clin Risk Manag. 2005;1(3):189-99.

41. Whitehead L, Seaton P. The effectiveness of self-management mobile phone and tablet apps in long-term condition management: a systematic review. J Med Internet Res. 2016;18(5):e97.

42. Timmers T, Janssen L, Kool RB, Kremer JA. Educating patients by providing timely information using smartphone and tablet apps: systematic review. J Med Internet Res. 2020;22(4):e17342.

43. DeFulio A, Silverman K. The use of incentives to reinforce medication adherence. Prev Med. 2012;55 Suppl(Suppl):S86-94.

44. Park KT, Ehrlich OG, Allen JI, et al. The cost of inflammatory bowel disease: an initiative from the Crohn's \& Colitis Foundation. Inflamm Bowel Dis. 2020;26(1):1-10.

45. Afzali A, Dalal S, Griffith J, Guntaka S, Padilla B, Wegrzyn L. Sa1745 Impact of a patient support program on inflammatory bowel disease-related hospitalization in patients treated with adalimumab. Presented at: Digestive Disease Week (DDW) Annual Meeting, 2020; Chicago, IL. 